\title{
A comparative study of Watson for Oncology and tumor boards in breast cancer treatment
}

\author{
Dohoon Kim ${ }^{1}$, Yun Young Kim ${ }^{1,2}$, Joon-Hyop Lee ${ }^{1,2}$, Yoo Seung Chung ${ }^{1,2}$, Sangtae Choi ${ }^{1,2}$, Jin Mo Kang ${ }^{1,2}$, Heung Kyu Park ${ }^{1,2}$, \\ Yong Soon Chun ${ }^{1,2}$ \\ 'Department of Surgery, Gachon University Gil Medical Center, Incheon; \\ ${ }^{2}$ Department of Surgery, Gachon University College of Medicine, Incheon, Korea
}

Purpose: Watson for Oncology (WFO) is a computing system for considering treatment option with patients. The aim of this study is to determine the concordance rate of WFO and tumor board in treatment options of breast cancer.

Methods: One hundred and seventy breast cancer patients who were treated at Gachon University Gil Medical Center (GMC) from December 2016 to March 2018 were investigated. "Concordance" is defined that treatment provided by GMC tumor board and WFO-provided treatments (Recommend or For Consideration) were coincide. "Discordance" is defined that treatment provided by GMC tumor board and WFO-provided treatments (Not Recommended or Not Available) were coincide.

Results: In chemotherapy, of the total 170 patients, 23 patients were excluded, and the concordance rate was compared in 147 patients. Concordance rate was 93\% (136/147). In the 11 patients who were discordant, seven patients complied with the treatment proposed by GMC tumor board due to insurance problems and age, and two patients did not follow the treatment proposed by WFO due to pregnancy and patient's choice, and one patient chose a treatment determined by WFO, last one patient was not treated with chemotherapy because it was not considered to be clinically necessary. In radiotherapy, the overall agreement rate was as high as 99\% (147/148).

Conclusion: Through this study, we found that many of the treatment proposals provided by WFO are highly reliable. Although the overall agreement is high in the radiotherapy regimen, the indication for radiotherapy by WFO is more aggressive and hypofractionated high dose intensity radiotherapy is growing trend by WFO.

Keywords: Watson for Oncology, Breast neoplasms, Treatment option, Concordance, Tumor board

Received: Jun 09, 2019 Revised: Jun 21, 2019 Accepted: Jun 22, 2019

Correspondence to: Yong Soon Chun

Department of Surgery, Gachon University Gil Medical Center, Gachon

University College of Medicine, 21 Namdong-daero 774beon-gil,

Namdong-gu, Incheon 21565, Korea

Tel: +82-32-458-2731, Fax: +82-32-460-3247

E-mail: chunysmd@gachon.ac.kr

ORCID: Dohoon Kim (https://orcid.org/0000-0002-6860-3508), Yun Young Kim (https://orcid.org/0000-0002-0077-540X), Joon-Hyop Lee (https://orcid.org/00000003-0470-7719), Yoo Seung Chung (https://orcid.org/0000-0001-9912-051X), Sangtae Choi (https://orcid.org/0000-0002-2074-1733), Jin Mo Kang (https:// orcid.org/0000-0002-1477-9778), Heung Kyu Park (https://orcid.org/0000-00028284-9221), Yong Soon Chun (https://orcid.org/0000-0002-7094-677X)

*This article was presented at 70th Annual Congress of the Korean Surgical Society 2018, on November 1-3, 2018, in Seoul, Korea.

Copyright (C) 2019 Korean Society of Surgical Oncology

This is an Open Access article distributed under the terms of the Creative Commons Attribution Non-Commercial License (http://creativecommons.org/licenses/by-nc/4.0) which permits unrestricted non-commercial use, distribution, and reproduction in any medium, provided the original work is properly cited.

\section{INTRODUCTION}

The treatment methods for breast cancer are evolving rapidly. New therapies such as radiation therapy, chemotherapy, and endocrine therapy, have been developed with the improvements in science and technology, and now molecular genetic methods have been developed to greatly affect the treatment and prognosis of breast cancer. As of October 2017, there were 69 drugs approved by the Food and Drug Administration for breast cancer treatment [1].

In a situation where technology in various fields is developed [2], the guidelines for the diagnosis and treatment of breast cancer and the development of treatment plans are improving at an accelerating rate [3]. Therefore, it is necessary to choose a treatment that suits the individual characteristics of a patient, but there are too many factors for a physician to synthesize and determine the knowledge in all fields.

Clinical decision-support systems can be a great solution to 
solve these difficulties because they can help collect and analyze patient information as well as assist the clinicians in treatment decisions [4].

Watson for Oncology (WFO) is a cognitive computing system designed by Memorial Sloan Kettering clinicians and IBM that considers various treatment options with patients. The system uses natural language, evaluates cases with evolving machine-learned models, and processes large volumes of data rapidly [5]. When WFO was introduced to Gachon University Gil Medical Center (GMC) in September of 2016, it had already learned more than 300 medical journals, more than 200 medical textbooks, and 1,500 pages of medical information [6]. These characteristics can be of great help in presenting a range of treatments for patients.

Because of the benefits of WFO, WFO is now being used to assist in the treatment of cancer patients at GMC. This study examined whether WFO can be an alternative to clinically useful treatment options by investigating the concordance rate between WFO and GMC tumor boards for patients with breast cancer.

\section{METHODS}

\section{Patients}

A total of 170 patients treated with breast cancer from December 2016 to March 2018 at GMC were included. GMC tumor boards composed of six multidisciplinary doctors using WFO discussed all patients, and treatment options presented by WFO and GMC tumor boards were compared. If the treatment options of WFO and GMC tumor boards are not consistent, treatment methods are determined by a discussion of members of GMC tumor board to reflect patient's preference. In 170 patients, 14 patients were excluded. Six patients refused chemotherapy and radiotherapy and histology results of four patients were unavailable from WFO. Two patients were diagnosed of other cancers in the past and WFO does not cover the patients with multiple cancers. One patient was lost in follow up and one case was required neoadjuvant chemotherapy but the patient requested surgery first. All patients had ductal, lobular and mixed histology of breast cancer. In 156 pa-

Table 1. Patient characteristics

\begin{tabular}{lc}
\hline Characteristic & Patient, no (\%) \\
\hline Total no. & 156 \\
Age, mean, yr & 52.65 \\
Stage & \\
I & $68(43)$ \\
II & $69(43)$ \\
III & $10(7)$ \\
Neoadjuvant & $9(7)$ \\
\hline
\end{tabular}

tients, 147 patients underwent adjuvant chemotherapy after surgical treatment, and nine patients experienced neoadjuvant chemotherapy.

\section{Concordance determination}

WFO suggests treatment options that consist of three categories (Recommend, For Consideration, and Not Recommended). In this study, "concordance" was defined as when the treatment options provided by GMC tumor boards and WFO-provided treatment options (Recommend or For Consideration) coincide. "Discordance" was defined as when the treatment options provided by GMC tumor boards and WFO-provided treatment options did not coincide (Not Recommended or Not Available). The concordance rate was only for chemotherapy and radiotherapy, not endocrine therapy.

\section{RESULTS}

Table 1 lists the patients' characteristics.

\section{Chemotherapy}

The Oncotype Dx test is an important factor in determining the treatment plan for chemotherapy in WFO. On the other hand, GMC has performed the Endopredict test instead of Oncotype Dx. Therefore, Oncotype Dx and Endopredict tests were assessed as they are the same. Among the 147 patients who received adjuvant chemotherapy, the concordance rate was 93\% (136/147) (Fig. 1). Six patients were not concordant due to the insurance problems. One patient who was not concordant was recommended CMF (cyclophosphamide+methotrexate $+5-\mathrm{FU}$ ) regimen by WFO but chemotherapy was not performed due to old age and history of heart disease. One patient with stage I breast cancer was not

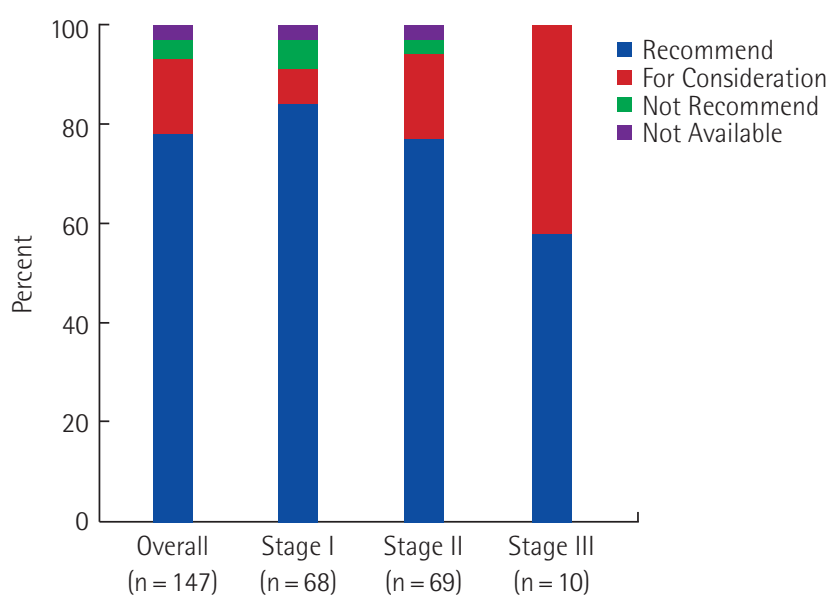

Fig. 1. Concordance rate in chemotherapy. 
Table 2. Discordant cases in chemotherapy

\begin{tabular}{|c|c|c|c|c|c|c|c|}
\hline Case & Stage & Pathology & ER/PR/her2 & WFO & GMC & Implement & Cause \\
\hline 1 & T1N0Mo & IDC & $+1-1+$ & $\mathrm{TH}+\mathrm{H}$ & $\mathrm{CMF}+\mathrm{H}$ & $\mathrm{CMF}+\mathrm{H}$ & Insurance \\
\hline 2 & T1NOMO & IDC & $+1+1+$ & $\mathrm{AC}+\mathrm{T}+\mathrm{H}$ & $\mathrm{CMF}+\mathrm{H}$ & $\mathrm{CMF}+\mathrm{H}$ & Insurance \\
\hline 3 & T1NOMO & IDC & $-1-1+$ & $\mathrm{TH}+\mathrm{H}$ & $\mathrm{CMF}+\mathrm{H}$ & $\mathrm{CMF}+\mathrm{H}$ & Insurance \\
\hline 4 & T1NOMO & IDC & $+1+1+$ & $\mathrm{TH}+\mathrm{H}$ & $\mathrm{CMF}+\mathrm{H}$ & $\mathrm{CMF}+\mathrm{H}$ & Insurance \\
\hline 5 & T2NOMO & ILC & $-|-|-$ & $\mathrm{AC}+\mathrm{T}$ & $\mathrm{CMF}$ & $\mathrm{CMF}$ & Insurance \\
\hline 6 & T1NOMO & IDC & $-|-|-$ & $\mathrm{AC}+\mathrm{T}$ & CMF & CMF & Insurance \\
\hline 7 & T1N0M0 & ILC & $+1+1-$ & CMF & No chemotherapy & No chemotherapy & Oncologist's opinion \\
\hline 8 & T1NOMO & IDC & $-|-|-$ & $\mathrm{CMF}$ & No chemotherapy & No chemotherapy & Oncologist's opinion \\
\hline 9 & T2NOMO & IDC & $+|-|-$ & $\mathrm{AC}+\mathrm{T}$ & CMF & No chemotherapy & Patient's choice \\
\hline 10 & T2NOMO & IDC & $-|-|-$ & $\mathrm{AC}+\mathrm{T}$ & $A C$ & $A C$ & Pregnancy \\
\hline 11 & T1N1M0 & IDC & $+1+1-$ & $\mathrm{AC}+\mathrm{T}$ & TC & $\mathrm{AC}+\mathrm{T}$ & Patient's choice \\
\hline
\end{tabular}

ER, estrogen receptor; PR, progesterone receptor; her2, human epidermal growth factor receptor 2; WFO, Watson for Oncology; GMC, Gachon University Gil Medical Center; IDC, invasive ductal carcinoma; ILC, invasive lobular carcinoma; TH, paclitaxel+trastuzumab; H, trastuzumab; AC, doxorubicin+cyclophosphamide; T, paclitaxel; CMF, cyclophosphamide+methotrexate+5-FU; TC, taxol+cyclophosphamide.

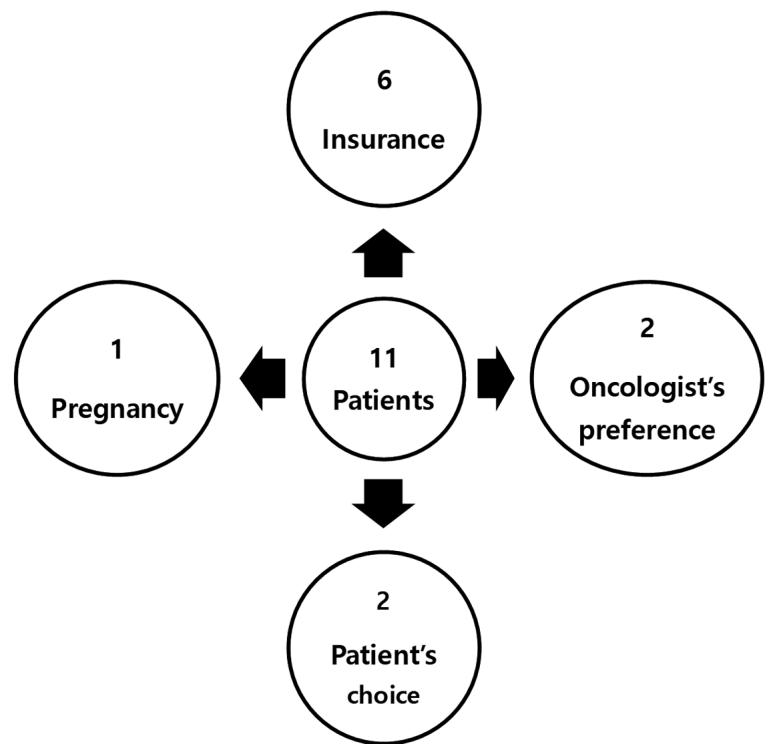

Fig. 2. Discordant cases in chemotherapy.

concordant because an oncologist did not recommend chemotherapy even though WFO recommended chemotherapy. Two patients were discordant due to patients' preference and one patient was not concordant because of pregnancy. Among the 11 inconsistent patients, nine patients chose the GMC treatment options rather than the WFO options, one selected WFO treatment option, and one refused chemotherapy (Table 2, Fig. 2).

Of a total 156 patients, nine patients underwent neoadjuvant chemotherapy, and seven of them had a concordance rate of $78 \%$ which is in agreement with the treatment options proposed by WFO and the GMC tumor boards. In nine cases of neoadjuvant chemotherapy, two cases were discordant with the proposal of
WFO and GMC tumor boards. WFO recommended palbociclib but due to weakly estrogen receptor (ER) positive, progesterone receptor (PR) negative, GMC oncologist recommended AC (doxorubicin+cyclophosphamide) followed by paclitaxel and the patients chose GMC treatment.

\section{Radiotherapy}

Radiotherapy as proposed by WFO has three methods: hypofractionated whole breast, conventionally fractionated whole breast with/without comprehensive regional nodes, and conventionally fractionated chest wall with comprehensive regional nodes. There are four types of radiotherapy in the GMC: breast only, breast with supraclavicular fossa, breast with supraclavicular fossa and posterior axilla boost, or breast with supraclavicular fossa, posterior axilla boost and internal mammary lymph nodes. Of the 155 patients, two patients failed to undergo radiotherapy due to follow-up loss after chemotherapy and nine patients had neoadjuvant chemotherapy. As a result, the data of 144 patients were examined.

The concordance rate of radiotherapy between WFO and GMC was 99\% (143/144); one patient was unable to perform radiotherapy due to pregnancy. The overall agreement rate was as high as 99\%, but there were some differences in the implementation. When hypofractionated whole breast radiotherapy was recommended by WFO, GMC proposed and implemented breast only radiotherapy. Adjuvant radiotherapy for patients with breast cancer in GMC was performed in patients with over T3 or N2 who had total mastectomy and with any state who underwent breast-conserving surgery. On the other hand, more aggressive radiotherapy is recommended by WFO, for example, radiotherapy is recommended to the patients who had mastectomy with any axil- 
lary lymph node metastasis or with severe lymphovascular invasion even without lymph node metastasis.

\section{DISCUSSION}

In this study, the concordance rate of chemotherapy was high. In the 11 patients who were not concordant, six patients complied with the treatment proposed by GMC due to insurance problems, and one patient complied with the GMC treatment because of old age. Another patient did not follow the treatment proposed by WFO due to pregnancy and one patient was not treated with chemotherapy because it was not considered to be clinically necessary. One patient selected the treatment option of WFO and one selected the GMC treatment options.

One of the drawbacks of comparing the chemotherapy concordance rates in this study was the synchronization of the recurrence risk. Oncotype Dx was required in WFO but we are using Endopredict test in GMC. Therefore, they were synchronized and used in the study [7]. According to a study comparing Endopredict test and Oncotype Dx risk groups, the agreement rate was moderate to $76 \%$. In addition, patients in a low-risk group of Oncotype Dx accounted for a high risk in Endopredict test in 18\% [8]. Since the risk group according to Endopredict test was synchronized with the risk group of Oncotype Dx, we could consider the high-risk patients by the Oncotype Dx were not downgraded to the low-risk patients. The problem should be resolved later if the WFO includes the Endopredict test in the patient information setting.

Compared to adjuvant chemotherapy, the concordance rate of neoadjuvant chemotherapy was lower than that of adjuvant chemotherapy because the chemotherapy regimens proposed by WFO in advanced cases are more not covered by insurance.

In the case of radiotherapy, hypofractionated whole breast radiotherapy was less frequently performed in GMC. Therefore, when the treatment of WFO and GMC were concordant, most cases were considered as "For Consideration" options proposed by WFO.

The WFO decision-making process, which presents the treatment options as soon as we input patient's data related to the disease could be more economically efficient, rather than the tumor board, which is composed of multiple doctors. However, the roles of WFO are a proposal for treatment and assisting a doctor. Future artificial intelligence services will be developed to fill doctor's deficiencies and make better choices. At present, the high concordance rate of GMC and WFO is not the ultimate success of artificial intelligence, but it shows the possibility of development of better artificial intelligence.

Through this study, we found most treatment options proposed by WFO were highly reliable. If the patient's personal information input is upgraded, it may be possible to obtain better results for individualized treatment. The weakness of this study is that the number of patients included in the study is small and the study period is short. Hence the recurrence rate could not be confirmed because the WFO had just started. Further studies considering these points will be needed.

\section{CONFLICT OF INTEREST}

No potential conflict of interest relevant to this article was reported.

\section{REFERENCES}

1. National Institutes of Health. Drugs approved for breast cancer [Internet]. Bethesda (MD): National Institutes of Health; c2019 [cited 2019 Jun 24]. Available from: http://www.cancer.gov/about-cancer/treatment/drugs/breast.

2. Taichman DB, Backus J, Baethge C, Bauchner H, de Leeuw PW, Drazen JM, et al. Sharing clinical trial data: a proposal from the international committee of medical journal editors. Ann Intern Med 2016;164:505-6.

3. Taichman DB, Backus J, Baethge C, Bauchner H, de Leeuw PW, Drazen JM, et al. Sharing clinical trial data: a proposal from the international committee of medical journal editors. $\mathrm{N}$ Engl J Med 2016;374:384-6.

4. Duda RO, Shortliffe EH. Expert systems research. Science 1983;220:261-8.

5. Somashekhar SP, Sepulveda MJ, Puglielli S, Norden AD, Shortliffe EH, Rohit Kumar C, et al. Watson for Oncology and breast cancer treatment recommendations: agreement with an expert multidisciplinary tumor board. Ann Oncol 2018;29:418-23.

6. International Business Machines Corp (IBM). Gil Hospital adopts IBM WFO for the first time in South Korea [Internet]. Seoul (KR): IBM News Release; c2017 [cited 2019 Jun 24]. Available from: http://www-03.ibm.com/press/kr/ko/pressrelease/50591.wss\#release.

7. Varga Z, Sinn P, Fritzsche F, von Hochstetter A, Noske A, Schraml P, et al. Comparison of EndoPredict and Oncotype DX test results in hormone receptor positive invasive breast cancer. PLoS One 2013;8:e58483.

8. Buus R, Sestak I, Kronenwett R, Denkert C, Dubsky P, Krappmann $\mathrm{K}$, et al. Comparison of EndoPredict and EPclin with oncotype DX recurrence score for prediction of risk of distant recurrence after endocrine therapy. J Natl Cancer Inst 2016;108:djw149. 